\title{
Performance of Several Bioequivalence Metrics for Assessing the Rate and Extent of Absorption
}

Husam A. Bayoud ${ }^{1 *}$ and Adnan M. Awad ${ }^{2}$

${ }^{1}$ Arts and Sciences Unit, Fahad Bin Sultan University, Tabuk, Kingdom of Saudi Arabia

${ }^{2}$ Department of Mathematics, University of Jordan, Amman, Jordan

\begin{abstract}
In this paper the problem of interest is the bioequivalence metrics and their role in assessing the rate and extent of absorption ratios of two drug formulations. Several bioequivalence metrics were proposed by several authors in the literature to estimate the similarity or dissimilarity of two drug formulations. The existing bioequivalence metrics are reviewed. A new bioequivalence metric is proposed and motivated. The performance, in terms of the statistical power, of the previously proposed and the new bioequivalence metrics is also evaluated by simulating cross-over bioequivalence trials.
\end{abstract}

Keywrds: Bioequivalence; Absorption rate; Absorption extent; Statistical power

\section{Introduction}

Bioavailability of a drug product is usually defined in terms of rate and extent to which the active drug ingredient is absorbed and becomes available at the site of drug action. Two drug formulations are said to be bioequivalent if their bioavailabilities are statistically similar.

Several measures, pharmacokinetic (PK) parameters, were proposed in the literature to estimate the bioavailability of a drug directly from the profile shape (concentration-time curve). The main PK parameters are: $A U C$ (area under profile shape), $C_{\max }$ (maximum concentration), $t_{\max }$ (time to the maximum concentration), $A U C_{P}$ (partial area under the profile shape from zero to $t_{\max }$ ). Bois et al. $[1,2]$ suggested to use $A U C$ and $C_{\max }$ to estimate the extent and rate of absorption of a drug formulation respectively. $C_{\max } / A U C$ and $C_{\max } / t_{\max }$ were recommended by Endrenyi and Tothfalusi [3] for the assessment of bioequivalence. Chen [4] suggested using the partial area under the blood concentration-time curve from zero to $t_{\max }$.

United States Food and Drug Administration (FDA) [5] suggested a typical criteria for the assessment of bioequivalence between test (T) and reference (R) drug formulations. According to this criteria, test and reference formulations are bioequivalent if boundaries of $90 \%$ confidence intervals for each of $A U C_{T} / A U C_{R}$ and $C_{\max }^{T} / C_{\max }^{R}$ lie between 0.8 and 1.25 .

It is not difficult to contrive a situation in which two different profiles yield identical $A U C, C_{\max }$ or $t_{\max }$. So, an analysis based on any one of these PK parameters may result in a determination that two drug formulations are bioequivalent when they are not in fact clinically equivalent. This is because these PK parameters do not take the profile shape in consideration. In view of this, a method that takes the profile shape into account may offer increased sensitivity to detect important differences between profiles. Such a method should depend on evaluating an appropriate distance measure to estimate the similarity or dissimilarity of two profiles. This distance measure is called bioequivalence metric.

Several authors have proposed different bioequivalence metrics for comparing two drug formulations over the entire profile. Most familiar bioequivalence metrics are reviewed. A simulated comparative study between those measures together with a new suggested metric is conducted.

\section{Most Familiar BE Metrics}

\section{Rescigno metric}

Rescigno [6] suggested a direct curve metric as a measure of dissimilarity between reference and test profiles, the general form for the Rescigno metric is:

$$
\xi_{j}=\left(\frac{\sum_{i=1}^{n}\left|C_{R}\left(t_{i}\right)-C_{T}\left(t_{i}\right)\right|^{j}}{\sum_{i=1}^{n}\left(C_{R}\left(t_{i}\right)+C_{T}\left(t_{i}\right)\right)^{j}}\right)^{\frac{1}{j}}
$$

where $C_{R}\left(t_{i}\right)=$ observed reference concentration at $t_{i}, C_{T}\left(t_{i}\right)=$ observed test concentration at $t_{i}$. Test and reference drug formulations are considered to be bioequivalent if the median observed Rescigno metric is less than or equal to 0.1 . In this paper, only $\xi_{1}$ and $\xi_{2}$ will be considered.

\section{Chinchilli metric}

Chinchilli and Elswick [7] proposed a different method for summarizing profile differences. Their approach is somewhat complex, but can be thought of as an accumulation of the pointwise relative differences between the two profiles. Roughly speaking, their metric measures the total relative difference, whereas Rescigno metric measures the relative total difference.

Assuming the \pm 20 rule, Chinchilli metric is estimated by using the trapezoidal rule as

$$
\psi=\frac{\sum_{i=1}^{n} 0.5\left(t_{i}-t_{i-1}\left\lfloor T_{U}\left(t_{i}\right)-T_{L}\left(t_{i}\right)+T_{U}\left(t_{i-1}\right)-T_{L}\left(t_{i-1}\right\rfloor\right.\right.}{\sum_{i=1}^{n} 0.5\left(t_{i}-t_{i-1}\left\lfloor R_{U}\left(t_{i}\right)-R_{L}\left(t_{i}\right)+R_{U}\left(t_{i-1}\right)-R_{L}\left(t_{i-1}\right\rfloor\right.\right.},
$$

*Corresponding author: Husam A. Bayoud, Arts and Sciences Unit, Fahad Bin Sultan University, Tabuk, Kingdom of Saudi Arabia, E-mail: husam.awni@yahoo. com

Received July 20, 2011; Accepted August 26, 2011; Published August 28, 2011

Citation: Bayoud HA, Awad AM (2011) Performance of Several Bioequivalence Metrics for Assessing the Rate and Extent of Absorption. J Bioequiv Availab 3 : 174-177. doi:10.4172/jbb.1000080

Copyright: (C) 2011 Bayoud HA, et al. This is an open-access article distributed under the terms of the Creative Commons Attribution License, which permits unrestricted use, distribution, and reproduction in any medium, provided the original author and source are credited. 
where $R_{L}\left(t_{i}\right)=0.8 \times C_{R}\left(t_{i}\right), R_{U}\left(t_{i}\right)=1.2 \times C_{R}\left(t_{i}\right), T_{L}\left(t_{i}\right)=\min \left[C_{T}\left(t_{i}\right), \frac{C_{R}\left(t_{i}\right)}{C_{T}\left(t_{i}\right)} C_{R}\left(t_{i}\right)\right]$

and $T_{U}\left(t_{i}\right)=\min \left[C_{T}\left(t_{i}\right), \frac{C_{R}\left(t_{i}\right)}{C_{T}\left(t_{i}\right)} C_{R}\left(t_{i}\right)\right] \cdot$

This metric can be interpreted as the ratio of the test region area to the reference region area; where the reference region is bounded by $R_{L}\left(t_{i}\right), R_{U}\left(t_{i}\right)$.

Test and reference drug formulations are considered to be bioequivalent if the median observed Chinchilli metric is less than or equal to 1 .

\section{Relative difference (f1) metric}

Moore and Flanner [8] suggested the following direct curve metric as a measure of dissimilarity between the reference and test profiles:

$$
f 1=\frac{\sum_{i=1}^{n}\left|C_{R}\left(t_{i}\right)-C_{T}\left(t_{i}\right)\right|}{\sum_{i=1}^{n} C_{R}\left(t_{i}\right)} \times 100
$$

Test and reference drug formulations are considered to be bioequivalent if the median observed $f 1$ is less than or equal to 20 .

\section{Polli and mclean metrics}

Polli and Mclean [9] suggested four bioequivalence metrics with their acceptance bioequivalence limits in order to compare the reference and test entire profiles, these metrics are $\left(\rho, \rho_{m}, \delta_{a}, \delta_{s}\right)$ which are defined below:

$$
\begin{aligned}
& \rho=\frac{\sum_{i=1}^{n}\left(C_{R}\left(t_{i}\right)+C_{T}\left(t_{i}\right)\right) \text { Ratio }\left(t_{i}\right)}{\sum_{i=1}^{n}\left(C_{R}\left(t_{i}\right)+C_{T}\left(t_{i}\right)\right)} \\
& \rho_{m}=\frac{\sum_{i=1}^{n}\left(C_{R}\left(t_{i}\right)+C_{T}\left(t_{i}\right)\right)\left(\operatorname{Ratio}\left(t_{i}\right)-1\right)}{\sum_{i=1}^{n}\left(C_{R}\left(t_{i}\right)+C_{T}\left(t_{i}\right)\right)} \\
& \delta_{a}=\frac{2 \sum_{i=1}^{n}\left|C_{R}\left(t_{i}\right)-C_{T}\left(t_{i}\right)\right|}{\sum_{i=1}^{n}\left(C_{R}\left(t_{i}\right)+C_{T}\left(t_{i}\right)\right)} \\
& \delta_{s}=\frac{4 \sum_{i=1}^{n} \frac{\left(C_{R}\left(t_{i}\right)-C_{T}\left(t_{i}\right)\right)^{2}}{\left(C_{R}\left(t_{i}\right)+C_{T}\left(t_{i}\right)\right)}}{\sum_{i=1}^{n}\left(C_{R}\left(t_{i}\right)+C_{T}\left(t_{i}\right)\right)}
\end{aligned}
$$

where $\operatorname{Ratio}\left(t_{i}\right)=\operatorname{Max}\left[\frac{C_{R}\left(t_{i}\right)}{C_{T}\left(t_{i}\right)}, \frac{C_{T}\left(t_{i}\right)}{C_{R}\left(t_{i}\right)}\right]$. If either $C_{R}\left(t_{i}\right)$ or $C_{T}\left(t_{i}\right)$ (but not both) equals zero, then a value of 10 is assigned to Ratio $\left(t_{i}\right)$. If both $C_{R}\left(t_{i}\right)$ and $C_{T}\left(t_{i}\right)$ are equal to zero, then a value of 1 is assigned to Ratio $\left(t_{i}\right)$.

Polli and Mclean [9] suggested 1.4, 0.35, 0.27 and 0.102 to be the upper acceptable limits for $\rho, \rho_{m}, \delta_{a}$ and $\delta_{s}$ respectively.

\section{Karalis and macheras metrics}

Karalis and Macheras [10] suggested the following three BE metrics:

$$
\begin{aligned}
& \text { MARD }=\frac{\sum_{i=1}^{n}|\lambda(i)-1|}{n} \\
& \text { MARD }_{w 1}=\frac{\sum_{i=1}^{n}\left(C_{R}\left(t_{i}\right)+C_{T}\left(t_{i}\right)\right)|\lambda(i)-1|}{\sum_{i=1}^{n}\left(C_{R}\left(t_{i}\right)+C_{T}\left(t_{i}\right)\right)} \\
& \text { MARD }_{w 2}=\frac{\sum_{i=1}^{n} \frac{t_{m}}{\left|t_{i}-t_{m}\right|+1}|\lambda(i)-1|}{n}
\end{aligned}
$$

where $\lambda(i)$ refers to $C_{R}\left(t_{i}\right) / C_{T}\left(t_{i}\right)$ or $C_{T}\left(t_{i}\right) / C_{R}\left(t_{i}\right)$ ratio and $t_{m}$ corresponds to the mean value of the time points where the maximum concentrations are observed for the test and reference formulations. The upper acceptable limit for these metrics is 0.20 .

\section{Proposed BE Metric}

A new direct curve comparison metric is suggested in order to assess the bioequivalence between test and reference drug formulations. This metric is defined as the percentage of the common area $(P C A)$ under test and reference concentration-time curves, which is given by:

$$
P C A=\frac{\int_{0}^{\infty} \min \left(C_{R}(t), C_{T}(t)\right) d t}{\int_{0}^{\infty} \max \left(C_{R}(t), C_{T}(t)\right) d t}
$$

where $C_{R}(t)$ and $C_{T}(t)$ are the reference and test concentration functions respectively.

$P C A$ metric can be estimated by using the trapezoidal rule as:

$$
P C A=\frac{\sum_{i=2}^{n}\left(t_{i}-t_{i-1}\right)\left(\min \left(C_{R}\left(t_{i-1}\right), C_{T}\left(t_{i-1}\right)\right)+\min \left(C_{R}\left(t_{i}\right), C_{T}\left(t_{i}\right)\right)\right)}{\sum_{i=2}^{n}\left(t_{i}-t_{i-1}\right)\left(\max \left(C_{R}\left(t_{i-1}\right), C_{T}\left(t_{i-1}\right)\right)+\max \left(C_{R}\left(t_{i}\right), C_{T}\left(t_{i}\right)\right)\right)}
$$

$P C A$ can be rewritten as:

$$
P C A=\frac{A U C^{*}}{A U C_{R}+A U C_{T}-A U C^{*}} \text {; where } A U C^{*} \text { is the area under }
$$
the region constructed by joining the points $\min \left(C_{R}\left(t_{i}\right), C_{T}\left(t_{i}\right)\right)$ for $i=1,2, \ldots, n$.

$$
\text { Consider the PK model } C(t)=\frac{F D C l}{V} \frac{k_{a}}{k_{a}-k_{e}}\left(e^{-k_{e} t}-e^{-k_{a} t}\right) \text {, }
$$

where $k_{a}, k_{e}, F, D, C l$, and $V$ are the first-order absorption, elimination rate constants, the extent of absorption, the dose, the clearance and the apparent volume of distribution, respectively. It can be easily shown that the area under concentration-time curve from zero to $L$ equals $F A \frac{k_{a}}{k_{a}-k_{e}}\left[\frac{1}{k_{a}}\left(e^{-k_{a} L}-1\right)-\frac{1}{k_{e}}\left(e^{-k_{e} L}-1\right)\right]$, where $A=\frac{D C l}{V}$. Using the second order Taylor expansions for $e^{-k_{a} L}$ and $e^{-k_{c} L}$, the above area can be approximated by $F A \frac{k_{a} L^{2}}{2}$. The labels $T$ and $R$ will be used to distinguish parameters of test and reference parameters in PK model. If test and reference formulations have the same $A$ and $F$, i.e. $A^{T}=A^{R}$ and $F^{T}=F^{R}$ then $P C A$ approximately 
Citation: Bayoud HA, Awad AM (2011) Performance of Several Bioequivalence Metrics for Assessing the Rate and Extent of Absorption. J Bioequiv Availab 3: 174-177. doi:10.4172/jbb.1000080

\begin{tabular}{|l|l|l|l|}
\hline Parameter & Distribution & Mean & Intra-subject CV \\
\hline Volume of Distribution, V & Normal & $1 \mathrm{~L} / \mathrm{kg}$ & $10 \%$ \\
\hline Clearence, CL & Normal & $0.374 \mathrm{~L} /(\mathrm{hr} \times \mathrm{kg})$ & $10 \%$ \\
\hline$k_{\alpha}$ Absorption rate constant, $k_{\alpha}$ & Normal & $1.39 \mathrm{hr}$ & $10 \%$ \\
\hline Bioavailability, F & Uniform & 0.5 & $0.1 \pm$ \\
\hline
\end{tabular}

Table 1: Distributions of Simulated PK Parameters.

\begin{tabular}{|c|c|c|c|c|c|c|c|c|c|c|c|}
\hline$k_{a}^{T} / k_{a}^{R}$ & $P C A$ & $\xi_{1}$ & $\xi_{2}$ & $f 1$ & Typical & $A \cup C_{p}$ & $\delta_{a}$ & $\delta_{s}$ & $\rho$ & MARD & $M A R D_{w 2}$ \\
\hline 1.00 & 100 & 100 & 100 & 100 & 100 & 100 & 100 & 100 & 100 & 100 & 100 \\
\hline 1.05 & 100 & 100 & 100 & 100 & 100 & 100 & 100 & 100 & 100 & 100 & 100 \\
\hline 1.1 & 100 & 100 & 100 & 100 & 100 & 100 & 100 & 100 & 100 & 100 & 100 \\
\hline 1.15 & 100 & 100 & 100 & 100 & 100 & 100 & 100 & 100 & 100 & 100 & 100 \\
\hline 1.2 & 100 & 100 & 100 & 100 & 100 & 100 & 100 & 100 & 100 & 100 & 100 \\
\hline 1.25 & 94 & 100 & 100 & 100 & 100 & 100 & 100 & 100 & 100 & 100 & 100 \\
\hline 1.3 & 20 & 100 & 100 & 100 & 100 & 100 & 100 & 100 & 100 & 100 & 100 \\
\hline 1.35 & 0 & 100 & 100 & 100 & 91 & 98 & 100 & 100 & 100 & 99 & 100 \\
\hline 1.4 & 0 & 100 & 99 & 100 & 20 & 15 & 100 & 100 & 100 & 87 & 100 \\
\hline 1.45 & 0 & 57 & 57 & 91 & 0 & 1 & 100 & 100 & 100 & 12 & 100 \\
\hline 1.5 & 0 & 0 & 0 & 0 & 0 & 0 & 99 & 67 & 100 & 0 & 100 \\
\hline 1.6 & 0 & 0 & 0 & 0 & 0 & 0 & 0 & 0 & 8 & 0 & 100 \\
\hline 1.7 & 0 & 0 & 0 & 0 & 0 & 0 & 0 & 0 & 0 & 0 & 100 \\
\hline 3.0 & 0 & 0 & 0 & 0 & 0 & 0 & 0 & 0 & 0 & 0 & 100 \\
\hline
\end{tabular}

Table 2: Statistical power in terms of $k_{a}^{T} / k_{a}^{R}$

\begin{tabular}{|c|c|c|c|c|c|c|c|c|c|c|c|}
\hline$F^{T} / F^{R}$ & $P C A$ & $\xi_{1}$ & $\xi_{2}$ & $f 1$ & Typical & $A \cup C_{p}$ & $\delta_{a}$ & $\delta_{s}$ & $\rho$ & MARD & $M A R D_{w 2}$ \\
\hline 1.00 & 100 & 100 & 100 & 100 & 100 & 100 & 100 & 100 & 100 & 100 & 100 \\
\hline 1.05 & 100 & 100 & 100 & 100 & 100 & 100 & 100 & 100 & 100 & 100 & 100 \\
\hline 1.1 & 100 & 100 & 100 & 100 & 100 & 100 & 100 & 100 & 100 & 100 & 100 \\
\hline 1.15 & 100 & 100 & 100 & 100 & 100 & 100 & 100 & 100 & 100 & 100 & 100 \\
\hline 1.2 & 100 & 100 & 100 & 100 & 100 & 100 & 100 & 100 & 100 & 100 & 100 \\
\hline 1.25 & 99 & 100 & 76 & 61 & 100 & 100 & 100 & 100 & 100 & 100 & 100 \\
\hline 1.3 & 42 & 0 & 0 & 0 & 0 & 95 & 100 & 100 & 100 & 99 & 100 \\
\hline 1.35 & 1 & 0 & 0 & 0 & 0 & 32 & 100 & 100 & 100 & 53 & 100 \\
\hline 1.4 & 0 & 0 & 0 & 0 & 0 & 0 & 11 & 100 & 100 & 0 & 100 \\
\hline 1.5 & 0 & 0 & 0 & 0 & 0 & 0 & 0 & 0 & 21 & 0 & 47 \\
\hline 1.6 & 0 & 0 & 0 & 0 & 0 & 0 & 0 & 0 & 0 & 0 & 0 \\
\hline 1.7 & 0 & 0 & 0 & 0 & 0 & 0 & 0 & 0 & 0 & 0 & 0 \\
\hline 3.0 & 0 & 0 & 0 & 0 & 0 & 0 & 0 & 0 & 0 & 0 & 0 \\
\hline
\end{tabular}

equals $\frac{k_{a}^{T}}{k^{R}}$ when $k_{a}^{T}<k_{a}^{R}$, and approximately equals $\frac{k_{a}^{R}}{k^{T}}$ when $k_{a}^{R}<k_{a}^{T}$. This means that, $P C A$ totally depends on absorption rates.

It is interesting to note that:

If $C_{R}(t)<C_{T}(t)$ at all time points then $P C A=\frac{A U C_{R}}{A U C_{T}}$.

If $C_{T}(t)<C_{R}(t)$ at all time points then $P C A=\frac{A U C_{T}}{A U C_{R}}$.

If $C_{R}(t)=C_{T}(t)$ at all time points then $P C A=1$.

It can be seen from the definition of the proposed metric, $P C A$, that it ranges from zero to one; and it approaches one when the two formulations are totally similar.
Based on 1000 simulated data sets from the above PK model with at most $20 \%$ differences in test and reference concentrations ratio, it is observed that the $90^{\text {th }}$ percentile of the $P C A$ values is 0.82 . This number is considered as the lower bioequivalence limit for $P C A$ with $90 \%$ confidence level.

\section{Simulation of Bioequivalence Trials, Baseline Scenario}

The algorithm proposed by Bois et al. [1] is used to simulate bioequivalence trials from the model:

$$
C(t)=A \frac{k_{a}}{k_{a}-k_{e}}\left(e^{-k_{e} t}-e^{-k_{a} t}\right)
$$

The parameters and their inter-subject and intra-subject variations, are summarized in Table 1. The parameters are generally identical to those proposed by Bois et al. [1]. The parameters $\mathrm{V}, \mathrm{Cl}$ and $k_{a}$ are 
assumed to have normal distributions. The parameter $\mathrm{F}$ is assumed to have uniform distribution, $U(0.4,0.6)$. A standard statistical model of error and variability was used to the parameters and to the concentrations, for more details see Bois et al. [1].

1000 bioequivalence data sets with 24 subjects are simulated under various absorption rate and extent ratios $k_{a}^{R}$ and $F^{1} / F^{R}$ respectively. Various BE metrics were computed from the test and reference concentration profiles for each subject. The acceptance or rejection of bioequivalence was also detected in each $\mathrm{BE}$ trial.

\section{Results and Discussions}

The main objective of this section is to examine the performance of the bioequivalence metrics $P C A, \xi_{1}, \xi_{2}, f 1$, Typical, $A U C_{\mathrm{p}}$-ratio, $\rho$ , $\delta_{a}, \delta_{s}, M A R D$ and $M A R D_{w 2}$. The statistical power, the probability of rejecting the null hypothesis of bioInequivalence in a data set, of those bioequivalence metrics have been computed as a function in the ratio $k_{a}^{T} / k_{a}^{R}$ and $F^{T} / F^{R}$, separately.

For each series of values of $k_{a}^{T} / k_{a}^{R}$ (and $F^{T} / F^{R}$ ), 1000 clinical trials with 24 subjects were generated by Monte Carlo simulations. The median of each of the previously proposed and the new bioequivalence metrics is computed for the 1000 data sets.

The proportion of accepting bioequivalence of two formulations when their absorption rate (or extent) ratio is outside the regulatory range is called the consumer risk, and the proportion of rejecting bioequivalence when their absorption rate (or extent) ratio is inside the regulatory range is called the producer risk.

With no difference between the test and reference formulations one would want to conclude bioequivalence in $100 \%$ of the data sets. In contrast, with a $25 \%(20 \%)$ difference or more in rate (extent) of absorption, a value used in regulatory practice [1,2], bioequivalence should be declared in no more than $5 \%$ of the data sets (i.e., a $5 \%$ consumer risk).

Statistical powers, the proportion of rejecting the null hypotheses of bioInequivalence, of the underlying BE metrics are computed and reported in Table 2 when $k_{a}^{T} / k_{a}^{R}$ ratio is greater than one, holding $F^{T} / F^{R}$ equals one. Table 3 summarizes the statistical power of the $\mathrm{BE}$ metrics when $F^{T} / F^{R}$ ratio is greater than one, holding $k_{a}^{T} / k_{a}^{R}$ equals one. Metrics exhibiting small consumer and producer risks should be favorable.

Table 2 shows that in the case of bioequivalence, when $k_{a}^{T} / k_{a}^{R}$ is less than 1.25 , the $\mathrm{BE}$ metrics lead to the true conclusion of $\mathrm{BE}$ in terms of rate of absorption, which means that the producer risk for all $\mathrm{BE}$ metrics equals zero. So, the performance of the BE metrics will be assessed according to the consumer risk values. The consumer risks are far from $5 \%$ for a $k_{a}^{T} / k_{a}^{R}$ of 1.25 . The $5 \%$ level or less is reached at 1.35 when using $P C A, 1.45$ when using Typical and $A U C_{\mathrm{p}}$-ratio, 1.5 when using $\xi_{1}, \xi_{2}, f 1$ and $M A R D$, and 1.6 when using $\rho, \delta_{a}$ and $\delta_{s}$. It can be also seen that $M A R D_{w 2}$ is insensitive to the changes in $k_{a}^{T} / k_{a}^{R}$.

Table 3 shows that in the case of bioequivalence, when $F^{T} / F^{R}$ is

less than 1.25, the BE metrics lead to the true conclusion of $\mathrm{BE}$ in terms of extent of absorption, which means that the producer risk for all $\mathrm{BE}$ metrics equals zero. So, it is enough to compare the consumer risk values of the $\mathrm{BE}$ metrics to test their performance. The consumer risks are far from $5 \%$ for an F-ratio of 1.25 . The 5\% level or less is reached at 1.3 when using Typical, $\xi_{1}, \xi_{2}$ and $f 1,1.35$ when using $P C A, 1.4$ when using $A U C_{\mathrm{p}}$-ratio, and $M A R D, 1.5$ when using $\delta_{a}$ and $\delta_{s}$ and 1.6 when using $\rho$ and $M A R D_{w 2}$.

\section{Conclusions}

As discussed in this paper, several bioequivalence metrics were proposed by several authors for the assessment of bioequivalence of two drug formulations. This paper proposed a new bioequivalence metric for estimating the pharmacological similarity between profiles by estimating the percentage of common area under test and reference formulations; this metric is referred to as $P C A$. This metric equals, under some conditions, the rate and extent of absorption. Simulation studies showed that PCA, Typical, $A U C_{\mathrm{p}}$-ratio, $\xi_{1}, \xi_{2}, f 1$ and $M A R D$ almost have the same behavior in assessing the BE. It is also shown that PCA followed by the Typical criteria perform well and better than the other metrics when the bioequivalence is assessing in terms of rate of absorption. On another hand, Typical, $\xi_{1}, \xi_{2}$ and $f 1$ followed by PCA metrics perform well and better than the other $\mathrm{BE}$ metrics when the bioequivalence is assessing in terms of extent of absorption.

\section{Acknowledgment}

Authors would like to thank anonymous referee for the valuable comments that improved this article.

\section{References}

1. Bois F, Tozer T, Hauck W, Chen M, Patnaik R, et al. (1993) Bioequivalence: Performance of Several Measures of Extent of Absorption. Pharm Research 11: 715-722.

2. Bois F, Tozer T, Hauck W, Chen M, Patnaik R, et al. (1994) Bioequivalence: Performance of Several Measures of Rate of Absorption. Pharm Research 11 966-974.

3. Endrenyi L, Tothfalusi L (1995) Without extrapolation, $C_{\text {max }} / A U C$ is an effective metric in investigations of bioequivalence. Pharm Research 12: 937-942.

4. Chen $M L$ (1992) An alternative approach for assessment of rate of absorption in bioequivalence studies. Pharm Research 9: 1380-1385.

5. FDA Guidance (1992) Statistical Procedures for Bioequivalence Studies Using a Standard Two-Treatment Crossover Design. Rockville, MD: Division of Bioequivalence, Office of Generic Drugs, Center for Drug Evaluation and Research, Food and Drug Administration 1992.

6. Rescigno A (1992) Bioequivalence. Pharm Research 9: 925-928.

7. Chinchilli VM, Elswick RK (1997) The multivariate assessment of bioequivalence. Journal of Biopharmaceutical Statistics 7: 113-123.

8. Moore JW, Flanner HH (1996) Pharm Tech 20: 64-74

9. Polli JE, McLean AM (2001) Novel Direct Curve Comparison Metrics for Bioequivalence. Pharm Research 18: 734-741.

10. Karalis V, Machras P (2003) Pharmacodynamic considerations in bioequivalence assessment: comparison of novel and existing metrics. Eur Jour of Pharm Scie 19: 45-56. 